\title{
Determination of the Exciton Binding Energy Using Photothermal and Photoluminescence Spectroscopy
}

\author{
K. Strzałkowski · J. Zakrzewski • M. Maliński
}

Received: 16 July 2012 / Accepted: 11 December 2012 / Published online: 29 December 2012 (C) The Author(s) 2012. This article is published with open access at Springerlink.com

\begin{abstract}
In this paper, experimental photoluminescence (PL) and piezoelectric photothermal (PPT) spectra of selected II-VI binary crystals are presented and analyzed. The quantitative analysis of the photothermal spectra was performed using a modified and extended Jackson-Amer model. The values of the bandgap energies of investigated semiconductors were computed from the PT amplitude and phase spectra. From the temperature dependence of the exciton emission so-called "excitonic energy gaps" have been determined. It follows from the theory that the exciton binding energy is the difference of these two values of energy gaps derived from PPT and PL spectroscopy.
\end{abstract}

Keywords A2B6 - Binary crystals · Exciton binding energy · Piezoelectric detection $\cdot$ Semiconductors

\section{Introduction}

The II-VI semiconductors are very promising materials from the point of view of application in construction of visible radiation sources in green laser diodes, spintronics, photodetectors, and other applications in modern optoelectronics [1-3]. The binary crystals investigated in this work ( $\mathrm{ZnSe}, \mathrm{ZnTe}, \mathrm{CdS}$, and $\mathrm{CdTe}$ ) can be treated as starting materials for mixed solid solutions. It is very important from the application point of view, that ternary and quaternary II-VI compounds allow almost a smooth change of

K. Strzałkowski $(\varangle) \cdot$ J. Zakrzewski

Institute of Physics, Faculty of Physics, Astronomy and Informatics,

Nicolaus Copernicus University, Grudziadzka 5, 87-100 Torun, Poland

e-mail: skaroll@fizyka.umk.pl

M. Maliński

Department of Electronics and Computer Science, Technical University of Koszalin,

ul. Sniadeckich 2, 75-328 Koszalin, Poland 
the bandgap and lattice constant values [4] (bandgap and lattice constant engineering). Since light emitting devices often operate on excitonic emission, a knowledge of the binding energy and physics of the excitons are of primary importance. An exciton is a hydrogen-like system formed in semiconductors, where a hole and an electron are attracting each other. The binding energy of such a system is much smaller than in the case of a hydrogen atom mainly due to the relatively high value of a dielectric constant of the crystal. The result is a Wannier exciton [5], which has a radius larger than the lattice spacing. In general, the population of such electron-hole pairs and their properties depend on many factors and present a rather complicated nature. Simplifying, there are two main factors influencing excitons, namely, the quality of the crystal and temperature. Because excitons use to travel within the crystal and their binding energy is of the order of several meV, the lattice should be well ordered. In such a system, every defect becomes a potential center of a non-radiative recombination of the electronhole pair. Hence, observations of the excitonic emission can give some information on the quality of the crystal itself. On the other hand, a typical exciton emission of the binary II-VI compound, coming from an electron-hole radiative recombination, is strongly thermally quenched. Its intensity, in the range of temperatures from liquid helium to room temperature (RT), decreases three or even four orders of magnitude. Thus, a stronger binding energy results in a much higher radiation efficiency at RT.

In this work, the piezoelectric photothermal (PPT) method developed by Jackson and Amer [6] and modified by Malinski [7,8] was used in theoretical calculations and experiment as well. This technique is considered to be a direct method as the signal depends on the quantum yield of the non-radiative transitions in semiconductors. One can state that this is also a complementary technique to the absorption and photoluminescence (PL) spectroscopy.

The aim of this article is to propose a new method of determining the exciton binding energy using PL and piezoelectric photothermal spectra of the selected II-VI binary semiconductors. Binary compounds were used to test the proposed method, because the parameters of these materials are well known. Thanks to that, it is possible to compare obtained values of the exciton binding energy with the literature data. What is more, thermal parameters also needed for theoretical simulations can be taken from the literature, allowing further reduction of the uncertainty of the final results.

\section{Experimental Setup and Sample Preparation}

All the investigated crystals were grown from the melt by the high pressure ( $150 \mathrm{~atm}$ of argon) modified Bridgman method using high purity (99.995\%) powders of $\mathrm{ZnSe}$, $\mathrm{ZnTe}, \mathrm{CdS}$, and CdTe put in a graphite crucible. This method allows one to obtain crystal rods of about one centimeter in diameter and up to a few centimeters in length. To remove processing, dirt crystal rods were etched in hydrochloric acid and next cut into about $1 \mathrm{~mm}$ thick samples, which were not orientated along any crystallographic plane. The plates were mechanically ground and then polished with diamond paste. For PL measurements samples after polishing were additionally chemically etched in a solution of sulfuric acid (96\%), potassium dichromate, and water. Photothermal experiments presented in this paper were performed on the computer-controlled photoacoustic spectrometer with the piezoelectric detection. Samples were illuminated 
with an intensity-modulated beam of light of a xenon lamp (Cermax $300 \mathrm{~W}$ ) after passing through the prism monochromator. The piezoelectric signal was detected with a lock-in phase selective amplifier (Stanford SR 510) at RT in the rear experimental configuration [9]. The PL setup consisted of the laser (HeCd, $325 \mathrm{~nm}, 20 \mathrm{~mW}$ ); closedcycle helium cryostat with the sample; temperature controller (LakeShore 331); and the spectrometer (MicroHR Horiba Jobin Yvon) equipped with diffraction gratings, filter wheel, and the thermoelectrically cooled CCD camera (Synapse Horiba Jobin Yvon). All measurements were performed in the temperature range from $10 \mathrm{~K}$ to $295 \mathrm{~K}$ every $10 \mathrm{~K}$ and were computer controlled.

\section{Results}

Computations of the PPT spectra were performed for the optical absorption coefficient $\beta(E)$ spectrum for the photon energies below and above the energy gap $\left(E_{\mathrm{g}}\right)$ of the crystal, represented by, respectively,

$$
\begin{aligned}
& \beta(E)=\beta_{0} \exp \left(\frac{\left(E-E_{\mathrm{g}}\right) \gamma}{0.025}\right) \text { for } E<E_{\mathrm{g}} \\
& \beta(E)=A_{0}\left(E-E_{\mathrm{g}}\right)^{\frac{1}{2}} \text { for } E>E_{\mathrm{g}},
\end{aligned}
$$

where $\beta_{0}, \gamma$, and $A_{0}$ are parameters. Equation 1 describes an Urbach tail thermal broadening of the absorption band observed for all direct electron type transition semiconductors [10]. The second equation (Eq. 2) describes the absorption band connected with direct, band-to-band, electron transitions in semiconductors. For $\mathrm{ZnSe}$ and CdTe samples to properly fit theoretical data to experimental results, it was necessary to consider the surface-type optical absorption coefficient spectrum connected with the presence of surface defects in the form of the Gaussian distribution (3)

$$
\beta(E)=A_{1 / 2} \exp \left(-\frac{\left(E-E_{1 / 2}\right)^{2}}{\beta_{1 / 2}^{2}}\right),
$$

where $A_{1 / 2}$ and $\beta_{1 / 2}$ are parameters, and $E_{1 / 2}$ represents energy locations of defects. The necessity of such an assumption arises from results of previous studies carried out for II-VI binary crystals, as well as for mixed ternary and quaternary solid solutions [9,11-14]. In general, to interpret the PPT signal, one must consider the periodical temperature field generated in the sample caused by the absorption of the intensity modulated periodical optical radiation. For computations, both volume and surface absorptions must be taken into account. The piezoelectric signal $S$ can be then expressed by the following formula [7]:

$$
S \cong-\left(\frac{1}{l} \int_{0}^{l} T(x, \beta) \mathrm{d} x-\frac{6}{l^{2}} \int_{0}^{l}\left(\frac{l}{2}-x\right) T(x, \beta) \mathrm{d} x\right),
$$




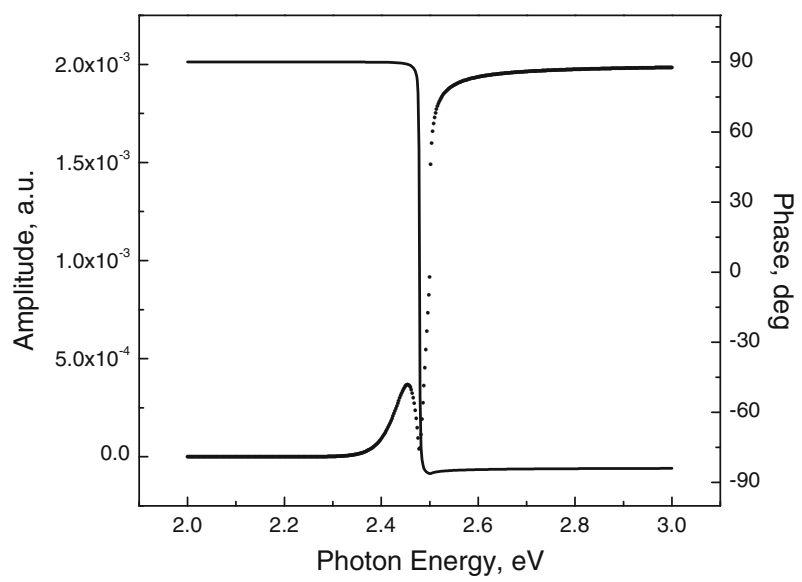

Fig. 1 Theoretical predictions for the amplitude (dots) and phase (line) spectra in the rear mode configuration for the following set of parameters: $\alpha=0.005 \mathrm{~cm}^{2} \cdot \mathrm{s}^{-1}, l=0.1 \mathrm{~cm}, f=36 \mathrm{~Hz}, E_{\mathrm{g}}=2.5 \mathrm{eV}, \gamma=1$

where $T(x, \beta)$ is the total temperature distribution field in the sample associated with the surface defects and volume absorption, $x$ is the spatial coordinate, and $l$ is the thickness of the sample. More details concerning theoretical aspects of a used model can be found in a previous paper [15].

Theoretical predictions for the amplitude and phase spectra in the rear measurement configuration are presented in Fig. 1. A characteristic peak in the sub-band gap region in the amplitude spectrum is clearly visible. According to the Jackson-Amer theory [6], the peak is due to subtracting the components originating from the piston-anddrum effect in the rear configuration mode. In the phase spectrum, this phenomenon is manifested as a change in the zero crossing value. However, it should be kept in mind that these theoretical spectra depend on many factors like the thickness of the sample, the modulation frequency, or the thermal diffusivity. What is more, for "real samples" the quality of the crystal, the preparation method of the samples [14], and the measurement setup are very important. Hence, a proper interpretation of real PPT spectra of investigated crystals can be often quite difficult even for pure binary compounds [13].

Figure 2 presents a theoretical fit of the theoretical amplitude and phase spectra to the experimental data for ZnSe (a, b), CdS (c, d), ZnTe (e, f), and CdTe (g, h) crystals, respectively. Parameters used for calculations were brought together in Table 1. The values of the thermal diffusivity and conductivity were taken from the literature [16]. All the spectra presented in Fig. 2 are typical for the rear mode configuration. In the amplitude for two cases (a) and (g), there are absorption bands visible in the sub-bandgap region connected with the surface absorption. Besides, in both cases for $\mathrm{ZnSe}$ and $\mathrm{CdTe}$, one can see the changes in the amplitude spectra. Only for CdTe is a change in the phase (h) also present. This can be explained on the basis of previous research. Only phase spectra are sensitive to the location of surface defects. It follows from the theory that a defect located on one side of the sample always affects the amplitude, but the phase only in a particular case [17]. 

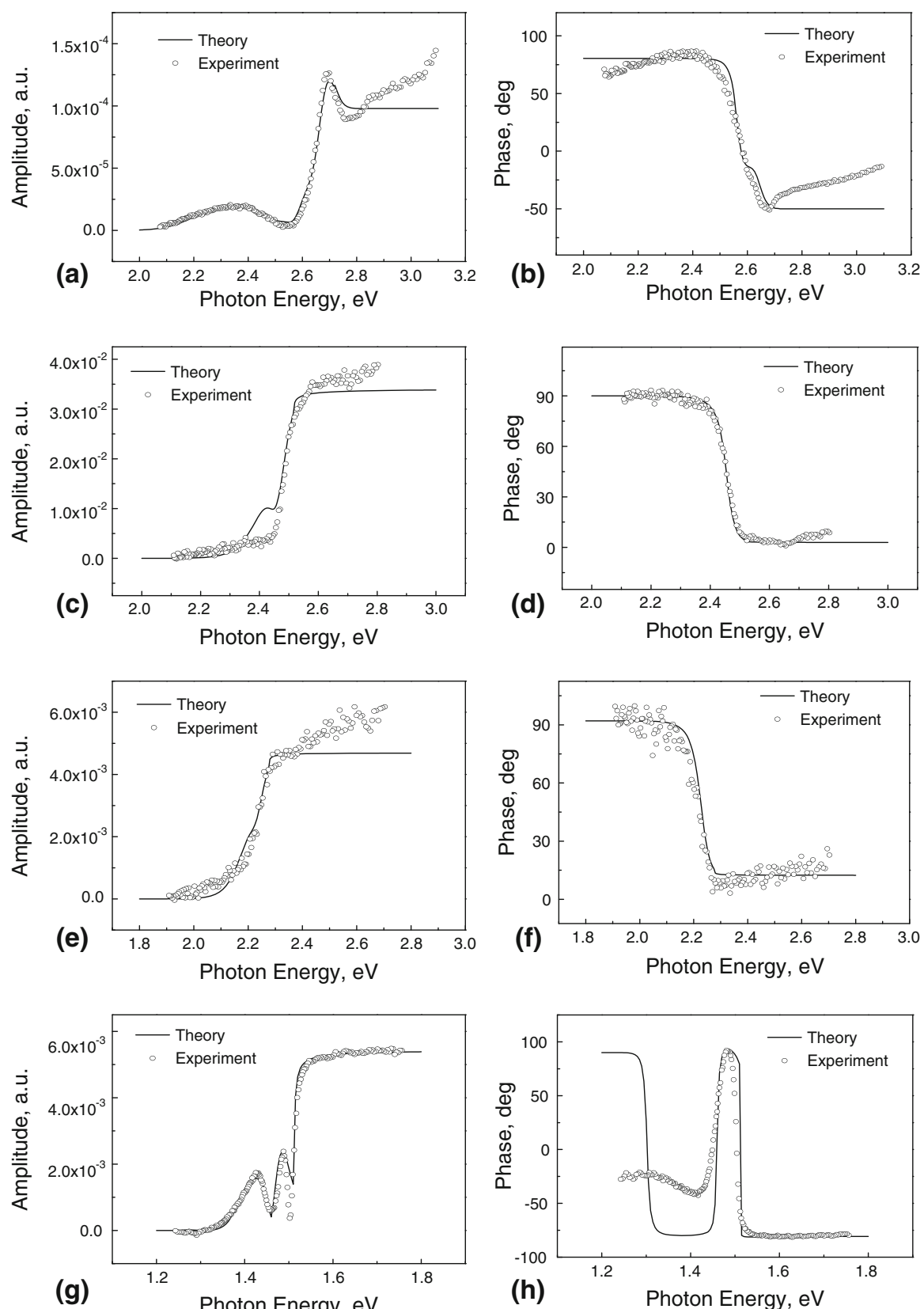

Fig. 2 Theoretical fit (line) to the experimental data (open circles) for (a, b) ZnSe, (c, d) CdS, (e, f) ZnTe, and $(\mathrm{g}, \mathrm{h}) \mathrm{CdTe}$ crystals for amplitude and phase spectra, respectively

A typical PL emission spectrum of a II-VI semiconductor is presented on the example of the $\mathrm{ZnSe}$ crystal at $40 \mathrm{~K}$ in Fig. 3. The spectrum consists of a high energy peak-exciton and so-called edge emission with a visible phonon structure just below 
Table 1 Summary of parameters used for calculations of the piezoelectric spectra of the researched crystals

\begin{tabular}{lllll}
\hline & ZnSe $(\mathrm{c})$ & CdS $(\mathrm{h})$ & ZnTe (c) & CdTe (c) \\
\hline$\beta_{0}\left(\mathrm{~cm}^{-1}\right)^{\mathrm{a}}$ & 100 & 100 & 100 & 50 \\
$\gamma\left(\mathrm{dimensionless}^{\mathrm{a}}\right.$ & 0.8 & 0.6 & 0.7 & 0.9 \\
$A_{0}\left(\mathrm{~cm}^{-1} \cdot \mathrm{eV}^{-0.5}\right)^{\mathrm{a}}$ & 5000 & 600 & 1000 & 1000 \\
$A_{1}, A_{2}\left(\mathrm{~cm}^{-1}\right)^{\mathrm{a}}$ & 200,2500 & - & - & 1200,500 \\
$E_{1}, E_{2}(\mathrm{eV})^{\mathrm{a}}$ & $2.33,2.65$ & - & - & $1.43,1.48$ \\
$\beta_{1}, \beta_{2}(\mathrm{meV})^{\mathrm{a}}$ & 160,55 & - & - & 45,20 \\
$E_{\mathrm{g}}(\mathrm{eV})^{\mathrm{a}}$ & 2.715 & 2.515 & 2.275 & 1.505 \\
$E_{\mathrm{g}}(\mathrm{eV})^{\mathrm{b}}$ & $2.69-2.73$ & $2.42-2.5$ & $2.25-2.29$ & $1.509-1.511$ \\
$\left.\alpha(\mathrm{cm})^{2} \cdot \mathrm{s}^{-1}\right)^{\mathrm{b}}$ & 0.1 & 0.15 & 0.2 & 0.05 \\
$\lambda\left(\mathrm{W} \cdot \mathrm{cm}^{-1} \cdot \mathrm{K}^{-1}\right)^{\mathrm{b}}$ & 0.19 & 0.2 & 0.18 & 0.01 \\
$l(\mathrm{~mm})^{\mathrm{c}}$ & 1.1 & 0.95 & 0.9 & 0.97 \\
$f(\mathrm{~Hz})^{\mathrm{c}}$ & 36 & 26 & 32 & 36 \\
\hline
\end{tabular}

${ }^{a}$ Parameters used for theoretical calculation of the amplitude and phase spectra

${ }^{b}$ Values taken from the literature [15] for cubic (c) and hexagonal (h) crystal structures

${ }^{\mathrm{c}}$ Experimental parameters

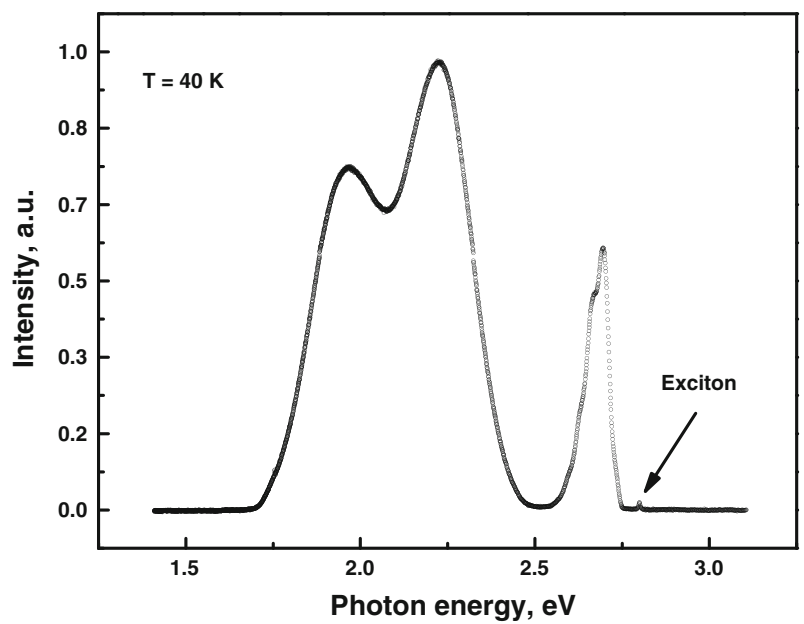

Fig. 3 Typical emission spectrum of a II-VI crystal for the example of ZnSe sample at $40 \mathrm{~K}$

the energy gap, which is connected with a radiative recombination of the carriers from shallow donor defects and a valence band, while deep defects are responsible for broadband(s), the last component in the long wave region of such a spectrum. The temperature evolution of the excitonic emission for the CdS sample in the temperature range from $10 \mathrm{~K}$ to $295 \mathrm{~K}$ is presented in Fig. 4. At low temperatures, two maxima positioned at $2.564 \mathrm{eV}$ and $2.571 \mathrm{eV}$ are observed in the spectrum. The maximum of the first high energy peak shifts monotonically to the lower energy with increasing temperature, and simultaneously temperature broadening is observed. This is a characteristic 


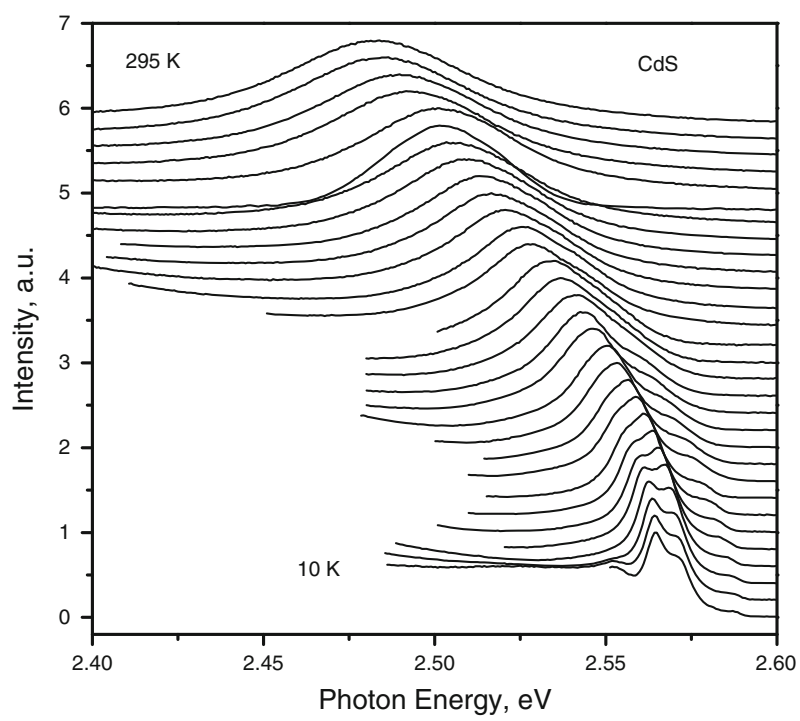

Fig. 4 Temperature evolution of the excitonic emission for CdS sample

behavior for free exciton recombination. The second maximum is thermally quenched at a temperature of about $80 \mathrm{~K}$ and is probably connected with recombination of some bound exciton, but the exact nature of this emission remains unknown. For other compounds, only free exciton and D-A recombinations were observed.

For all investigated crystals, the temperature dependence of the maximum of the excitonic emission was interpreted using the following Varshni formula:

$$
E_{\mathrm{g}}(T)=E_{\mathrm{g}}(0)-\frac{\gamma T^{2}}{\beta+T},
$$

where $E_{\mathrm{g}}(0)$ is the value of the bandgap at $0 \mathrm{~K}$. Constants $\gamma$ and $\beta$ are connected with the electron (exciton)-phonon interaction and Debye temperature, respectively. The best fits of the energetic position of the maximum of the excitonic emission expressed by the Varshni formula to the experimental data obtained from PL spectra are presented in Fig. 5. Parameters $\gamma$ and $\beta$ obtained from the fits remain in good agreement with the literature data [16]. Unfortunately, for the CdTe crystal, excitonic emission was not visible at RT. In this case, the energy of the position of the excitonic line at RT was obtained from Eq. 5. The final values of the exciton binding energy are collected in Table 2.

\section{Discussion}

Photoacoustic spectra were measured about ten times for each sample with a single step of $0.5 \mathrm{~nm}$ and a monochromator slit of $0.5 \mathrm{~mm}$. The values of the energy bandgaps obtained from theoretical calculations were averaged with a standard deviation of about 

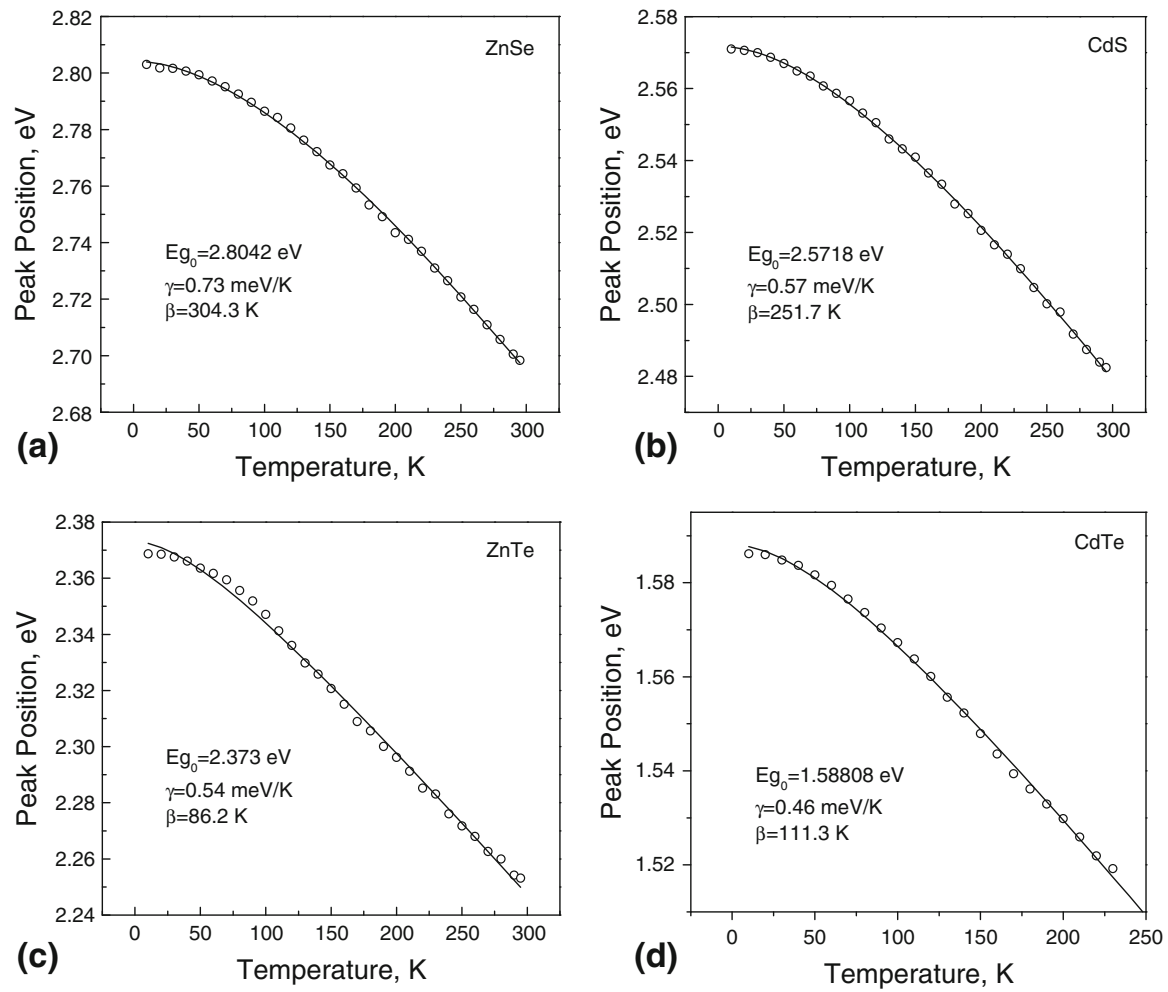

Fig. 5 Temperature dependences (open circles) of the maximum of excitonic emission for (a) ZnSe, (b) $\mathrm{CdS}$, (c) ZnTe, and (d) CdTe with theoretical fits (line) of a Varshni formula

Table 2 Final values of the exciton binding energy for the investigated crystals

\begin{tabular}{|c|c|c|c|c|c|}
\hline \multirow[t]{2}{*}{ Compound } & \multicolumn{3}{|c|}{ Bandgap at RT (eV) } & \multicolumn{2}{|c|}{ Exciton binding energy (meV) } \\
\hline & PL & PPT & Literature & Experiment & Literature \\
\hline $\mathrm{ZnSe}$ & 2.6983 & 2.715 & $2.69-2.73$ & 16.7 & $18-21$ \\
\hline $\mathrm{CdS}$ & 2.4825 & 2.515 & $2.42-2.5$ & 32.5 & 29 \\
\hline ZnTe & 2.2624 & 2.275 & $2.25-2.29$ & 12.6 & 13 \\
\hline $\mathrm{CdTe}$ & 1.49 & 1.505 & $1.509-1.511$ & 15 & 11 \\
\hline
\end{tabular}

$5 \mathrm{meV}$. Taking into account measurement conditions and theoretical simulation results, one can estimate the accuracy of determining the energy gap from PPT spectroscopy as about $7 \mathrm{meV}$ to $8 \mathrm{meV}$. For $\mathrm{ZnSe}$ and CdTe samples, theoretical calculations of energy gaps were more complicated than in the case of the other two semiconductors because surface absorption had to be considered. PL spectra of researched crystals were collected with a single step of $0.15 \mathrm{~nm}$ and a monochromator slit of $0.1 \mathrm{~mm}$ or smaller. For a well-defined excitonic line at RT, the uncertainty of determining its position in such a case is about $2 \mathrm{meV}$ to $3 \mathrm{meV}$. Hence, from PPT and PL spectroscopy, one can get 
the total uncertainty of determining the exciton binding energy as $9 \mathrm{meV}$ to $11 \mathrm{meV}$. It should be kept in mind, that in the case of the CdTe sample, the excitonic emission was not visible at RT and additional uncertainty must be taken into account. For the CdS crystal, the value of the binding energy was the highest of all and was equal to $32.5 \mathrm{meV}$ ( $29 \mathrm{meV}$ from the literature). This is a very important result from the application point of view, because thanks to that, the excitonic line at RT is clearly visible and well defined. On the other hand, for the CdTe crystal, the obtained value of the binding energy was only $15 \mathrm{meV}$ ( $11 \mathrm{meV}$ from the literature), and as a consequence, the excitonic emission was thermally quenched already at about $220 \mathrm{~K}$ to $230 \mathrm{~K}$.

\section{Conclusions}

PL and PPT spectra can be used to determine the exciton binding energy. Obtained values for investigated crystals remain in good agreement with the literature data (Table 2). The method is quite fast and simple and can be useful for samples where traditional transmission spectroscopy cannot be applied. The proposed method could be used when a sample of interest is quite thick, and as a consequence, the dynamics of the transmission measurement is too low to get the value of its energy gap properly. Another possibility includes crystals with absorption bands in the sub-bandgap region, just below the energy gap originating for the example from isoelectronic dopants such as manganese in $\mathrm{ZnMnSe}$ compounds. To apply this method, some conditions must be satisfied. First of all, the excitonic emission should be visible at RT to avoid additional uncertainty from the theoretical prediction of the position of the excitonic line using a Varshni formula. Surface states absorption can introduce further uncertainty. Hence, very important is the optimization of a sample preparation process to reduce these phenomena [14]. The main disadvantage of the proposed method is the quite large final uncertainty of the obtained results. However, it can be reduced through further optimization of the sample preparation, measurement, and theoretical calculation processes.

Open Access This article is distributed under the terms of the Creative Commons Attribution License which permits any use, distribution, and reproduction in any medium, provided the original author(s) and the source are credited.

\section{References}

1. T. Nakamura, K. Katayama, H. Mori, S. Fujiwara, Phys. Status Solidi B 241, 2659 (2004)

2. M. Adachi, K. Ando, T. Abe, N. Inoue, A. Urata, S. Tsutsumi, Y. Hashimoto, H. Kasada, K. Katayama, T. Nakamura, Phys. Status Solidi B 243, 943 (2006)

3. Y. Niiyama, M. Watanabe, Semicond. Sci. Technol. 20, 1187 (2005)

4. F. Rozpłoch, J. Patyk, F. Firszt, S. Łęgowski, H. Męczynska, J. Zakrzewski, A. Marasek, Phys. Status Solidi B 229, 207 (2002)

5. G. Wannier, Phys. Rev. 52, 191 (1937)

6. W. Jackson, N.M. Amer, J. Appl. Phys. 51, 3343 (1980)

7. M. Maliński, Arch. Acoust. 28, 43 (2003)

8. M. Maliński, Mol. Quantum Acoust. 23, 277 (2002)

9. M. Maliński, J. Zakrzewski, K. Strzałkowski, Int. J. Thermophys. 28, 299 (2007) 
10. F. Urbach, Phys. Rev. 92, 1324 (1953)

11. M. Maliński, J. Zakrzewski, Eur. Phys. J. Spec. Top. 154, 345 (2008)

12. M. Maliński, J. Zakrzewski, K. Strzałkowski, S. Łęgowski, Acta Acustica United Acustica 94, 250 (2008)

13. Ł. Chrobak, M. Maliński, J. Zakrzewski, K. Strzałkowski, Surf. Sci. 603, 3282 (2009)

14. J. Zakrzewski, M. Maliński, K. Strzałkowski, F. Firszt, S. Łęgowski, H. Męczyńska, Int. J. Thermophys. 31, 208 (2010)

15. J. Zakrzewski, M. Maliński, K. Strzałkowski, D. Madaj, F. Firszt, S. Łęgowski, H. Męczyńska, Int. J. Thermophys. 33, 733 (2012)

16. I. Hernández-Calderón, in II-VISemiconductor Materials and Their Applications, ed. by M.C. Tamargo (Taylor and Francis, New York, 2002), p. 113

17. M. Maliński, J. Zakrzewski, K. Strzałkowski, S. Łęgowski, F. Firszt, H. Męczyńska, Surf. Sci. 603, 131 (2009) 